\title{
Multiple HOM-C gene interactions specify cell fates in the nematode central nervous system
}

\author{
Stephen J. Salser, Curtis M. Loer, and Cynthia Kenyon \\ Department of Biochemistry and Biophysics, University of California, San Francisco, California 94143-0554 USA
}

\begin{abstract}
Intricate patterns of overlapping HOM-C gene expression along the $\mathrm{A} / \mathrm{P}$ axis have been observed in many organisms; however, the significance of these patterns in establishing the ultimate fates of individual cells is not well understood. We have examined the expression of the Caenorhabditis elegans Antennapedia homolog mab- 5 and its role in specifying cell fates in the posterior of the ventral nerve cord. We find that the pattern of fates specified by mab-5 not only depends on mab-5 expression but also on post-translational interactions with the neighboring HOM-C gene lin-39 and a second, inferred gene activity. Where mab-5 expression overlaps with lin-39 activity, they can interact in two different ways depending on the cell type: They can either effectively neutralize one another where they are both expressed or lin-39 can predominate over mab-5. As observed for Antennapedia in Drosophila, expression of mab-5 itself is repressed by the next most posterior HOM-C gene, egl-5. Thus, a surprising diversity in HOM-C regulatory mechanisms exists within a small set of cells even in a simple organism.
\end{abstract}

[Key Words: Caenorhabditis elegans; anterior-posterior axis; homeotic cluster; mab-5; combinatorial interactions; homeo box]

Received May 25, 1993; revised version accepted July 8, 1993.

Organisms as diverse as insects, vertebrates, and nematodes use a conserved system of Antennapedia-class homeo box genes to pattern the anterior-posterior $(\mathrm{A} / \mathrm{P})$ axis (for review, see McGinnis and Krumlauf 1992; also see Clark et al. 1993; Wang et al. 1993). These homeotic complex (HOM-C) genes are expressed in restricted domains along the $\mathrm{A} / \mathrm{P}$ axis and are thought to act as transcriptional regulators to give different regions of the body their identities.

In insects, where the metameric organization of the body is especially striking, HOM-C genes are often thought of as acting to specify parasegment identities. Consistent with this view, abrupt changes in HOM-C gene expression correspond to a first approximation with parasegment boundaries (Duncan 1986; Ingham and Martinez-Arias 1986; Carroll et al. 1988). Moreover, several HOM-C genes cause near complete specification of particular segmental identities when expressed ectopically under the control of heat shock promoters (Gibson and Gehring 1988; Kuziora and McGinnis 1988; González-Reyes and Morata 1990; Mann and Hogness 1990). Thus, despite the fact that domains of HOM-C gene expression overlap along the $\mathrm{A} / \mathrm{P}$ axis, in many cases, a single gene is largely responsible for the identity of a particular parasegment (see, however, Struhl 1982).

This basic model (one gene, one parasegment identity) helps to describe the role of HOM-C genes in patterning whole body parts. At the level of single cells, however, expression of these genes is surprisingly complicated. Within parasegments, cells expressing a given HOM-C gene are interspersed with nonexpressing cells and with cells expressing other HOM-C members (Beachy et al. 1985; White and Wilcox 1985; Carroll et al. 1986; Wirtz et al. 1986). In some cases, particularly in the central nervous system (CNS), specific neurons maintain strong coexpression of multiple HOM-C genes (White and Wilcox 1985; Carroll et al. 1986,1988; Celniker et al. 1990; Karch et al. 1990). These expression patterns raise several questions. To what extent does HOM-C gene expression directly specify the domain of gene function? Moreover, in cells expressing multiple HOM-C genes, what type of functional interactions can take place? We have taken advantage of the reproducible cell lineage and simplicity of Caenorhabditis elegans to explore these questions with single-cell resolution.

A cluster of four HOM-C genes has been identified in C. elegans (Schaller et al. 1990; Bürglin et al. 1991; Kenyon and Wang 1991). Mutations in three of the genes, lin-39, mab-5, and egl-5, affect development in sequential regions of the body along the $\mathrm{A} / \mathrm{P}$ axis (Kenyon 1986; Chisholm 1991; Clark et al. 1993; Wang et al. 1993). These genes resemble the Deformed/Sex-combs-reduced, Antennapedia/Ultrabithorax (Ubx)/abdominal$A$, and $A b d o m i n a l-B(A b d-B)$ genes in Drosophila and are arranged similarly within the cluster. In contrast to previous reports (Bürglin et al. 1991; Kenyon and Wang 
1991), the C. elegans genome sequencing project has recently found ceh-13, a labial homolog, to lie between lin-39 and mab-5 suggesting that part of the C. elegans cluster has been inverted (R. Waterston, pers. comm.).

The $C$. elegans gene mab-5 is most closely related to Drosophila Antennapedia and is needed to specify the fates of many neuronal, epidermal, and mesodermal cells in a posterior body domain (Kenyon 1986; Costa et al. 1988). We have examined the role of $m a b-5$ in patterning the ventral ectoderm, which derives from 12 precursor cells (P1-P12; see Fig. 1A). During the first larval stage, these cells divide in a stereotypical pattern to contribute neurons to the ventral nerve cord. Each $P$ cell gives rise to one epidermal cell and five neuronal cells with distinct fates (Sulston and Horvitz 1976). The HOM-C genes create variations in this basic pattern of fates, causing cells in specific body regions to adopt specialized fates. In the posterior of the male, mab-5 is required to specify the proper fates of three sets of P-cell descendants: the Pn.p cells, which adopt epidermal fates, the Pn.aap cells, which produce sex-specific neurons, and the Pn.aaap cells, which become motoneurons (Fig. 1B).

If $m a b-5$ activity were simply restricted to the posterior P-cell lineages and acted as a switch to turn on mab5-dependent specializations, one might expect to see a contiguous block of specialized cells in the posterior; however, this is not the observed pattern. The boundary between specialized cells and more anterior cells occurs in different positions along the $\mathrm{A} / \mathrm{P}$ axis for each of the three decisions controlled by mab-5 (Fig. 1C). Five Pn.p cells are affected by mab-5, but only three Pn.aap cells and only two Pn.aaap cells are affected.

How could mab-5 be regulated to produce such a complex pattern? One possibility is that the expression of mab-5 itself is complex. mab-5 could be expressed specifically in those cells that adopt mab-5-dependent specializations and not in more anterior cells. In this case, mab-5-expressing and -nonexpressing cells would necessarily be interspersed with one another (Fig. 1D). Alternatively, mab-5 could be expressed in a graded fashion, and cells could decide whether to adopt specialized fates based on the level of mab-5. Finally, it is possible that mab-5 is expressed in a simple block of cells in the posterior. The boundary of mab-5 expression could then determine one of the boundaries between specialized and more anterior cell fates and the other boundaries could arise through interactions with other genes.

To distinguish between these models we have examined the mab-5 expression pattern, as well as the effects of expressing mab-5 ectopically. We find that Mab-5 pro-
A

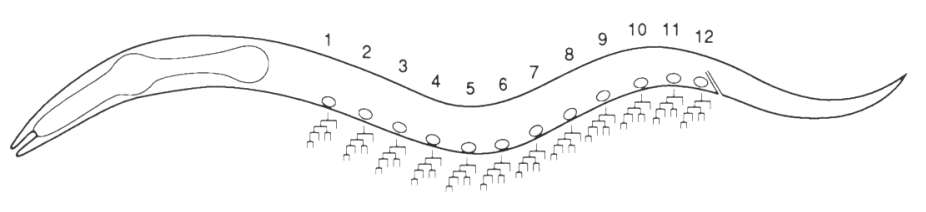

C

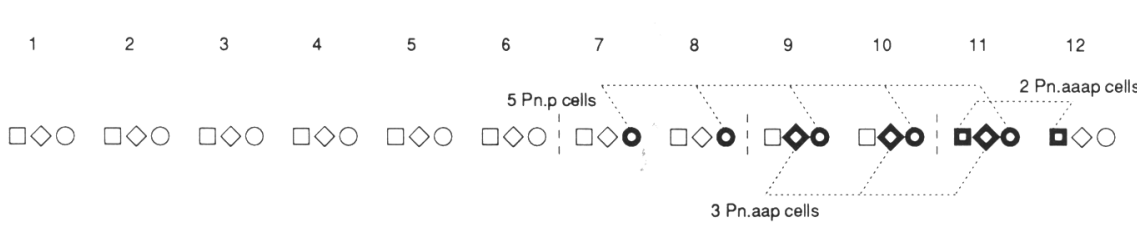

D

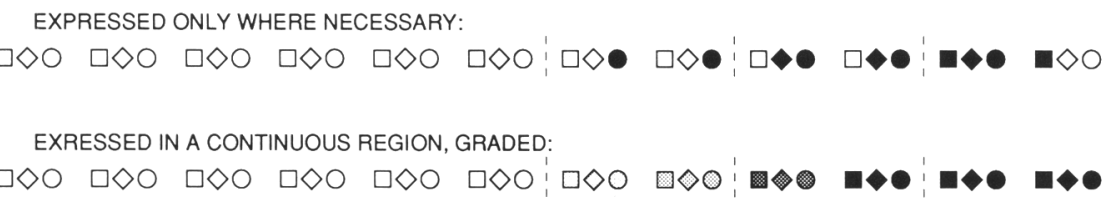

EXPRESSED IN A CONTINUOUS REGION, OTHER GENES ALSO INVOLVED:

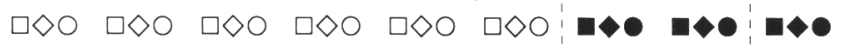

gene X

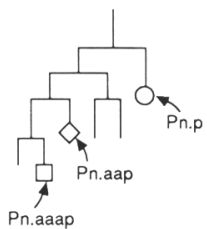
along the $\mathrm{A} / \mathrm{P}$ axis. $(B)$ Individual $\mathrm{P}$-cell lineage, showing the three types of descendants influenced by mab-5 in the male. The Pn.p cell is epidermal and requires mab-5 to avoid fusion with the surrounding epidermal syncytium. The Pn.aap cell is a sex-specific neuronal cell and requires mab-5 to divide. The Pn.aaap cell is a VB type motoneuron, except in $\mathrm{P}(11,12)$ lineages, where it requires mab-5 to undergo programmed cell death. (C) Schematic diagram showing the overall pattern of $P$ descendants that require mab-5 (in bold). Five Pn.p, three Pn.aap, and two Pn.aaap cells require mab-5 to adopt their normal fates. The cells are represented by circles, diamonds, and squares, respectively, and are derived from the $P$ lineages indicated above ( $1-$ 12). (D) Three models for how mab-5 activity could be regulated to produce this pattern: (1) Expression of mab-5 itself is regulated so as to influence only

those cells that require mab-5. Expressing cells (filled) must be interspersed with nonexpressing cells (open). (2) Graded expression of mab-5 is interpreted using multiple thresholds to create multiple boundaries in cell fate. (3) mab-5 expression only determines one of the boundaries in cell fate. Other genes (X and $\mathrm{Y}$ ) act to create other boundaries in cell fate. Dotted lines mark the locations of the three anterior boundaries in cell fate. 
tein is present in a contiguous block of cells in the posterior ventral nerve cord, where it serves to define only one of the boundaries in cell fate. The other boundaries arise through interactions with the more anterior HOM-C gene lin-39 and an inferred gene activity in the posterior. The interactions between these genes vary depending upon the cell type: In the Pn.p cells, mab-5 and lin-39 effectively neutralize one another where they are both expressed; in contrast, in the Pn.aap cells, lin-39 activity is dominant over mab-5 activity; finally, in the Pn.aaap cells, mab-5 is not sufficient on its own but may instead cooperate with another gene activity. These interactions are sufficient to explain the complex anterior boundary of mab-5 activity.

Previous work demonstrates that the posterior boundary of mab-5 function is restricted by the HOM-C gene eg1-5 (Chisholm 1991): Normally, mab-5 mutations alter the $\mathrm{P}(7-11) . \mathrm{p}$ fates, whereas in an egl-5 background mab-5 mutations affect $\mathrm{P}(7-12)$.p. Consistent with this genetic interaction, we have found that egl-5 represses mab-5 expression in the P12 lineage.

\section{Results}

mab-5 is expressed in a contiguous region of the ventral cord

To get a basic understanding of how mab-5 is regulated to create differences along the $\mathrm{A} / \mathrm{P}$ axis, we first examined the pattern of $m a b-5$ expression at different times in development using polyclonal antibodies (Fig. 2A-D). Mab-5 protein was localized to the nuclei of many cells in the posterior, consistent with its role as a region-specific transcription regulator. We found detectable levels of Mab-5 in the posterior P lineages (P7-P12), but not in more anterior cells. Within the P7-P12 region, Mab-5 expression was graded with the highest levels in the P10 descendants. The descendants of any one $\mathrm{P}$ cell expressed comparable levels of Mab-5.

In newly hatched larvae, the $\mathrm{P}$ cells are not yet positioned ventrally but, instead, are located in two rows of six cells: one row on each side of the animal. In both sexes, Mab-5 protein was seen consistently in nuclei of $\mathrm{P} 9 / 10$ and $\mathrm{P} 11 / 12$ (Fig. $2 \mathrm{~B}$ ) but not in more anterior $\mathrm{P}$ cells. Mab-5 was also detected at high levels in the posterior juvenile motoneurons located ventrally (Fig. 2A), although its role in these cells is unknown.

Approximately $8 \mathrm{hr}$ after the time of hatching, the $\mathrm{P}$ cells descend into the ventral nerve cord and interdigitate to form a single row of 12 cells, P1-P12. In both sexes, we detected Mab- 5 in P7 and P8, as well as in P9-P12 (not shown). In slightly older larvae, in which some $\mathrm{P}$ cells had divided, Mab-5 was found consistently in P7-P12 descendants but not in more anterior P descendants (Fig. 2C).

The completion of these divisions establishes a row of P-derived neuronal cells interspersed with juvenile motoneurons and epidermal Pn.p cells. In the male, descendants of P10 consistently expressed high levels of Mab-5, those of $\mathrm{P} 9$ and P11 expressed lower levels, and those of
P7 and P8 expressed barely detectable levels (Fig. 2D). P12 descendants were found to express Mab-5 only until the division of P12.a.

The descendants of any one $\mathrm{P}$ cell expressed comparable levels of Mab-5, the only exceptions being the Pn.p cells, which have larger and more diffuse nuclei, and the Pn.aap cells, which consistently expressed lower levels than their neighbors. Expression levels in the P descendants were similar to those seen in the neighboring juvenile motoneurons: Both sets of cells expressed mab-5 in a graded pattern, tapering off toward the anterior.

The expression pattern in hermaphrodites was identical to that just described in males, with two exceptions: the $\mathrm{P}(9-11)$.aap cells, which undergo programmed cell death, and the $\mathrm{P}(9-11) . \mathrm{p}$ cells, which fuse with the epidermal syncytium. Both of these cell types no longer expressed mab-5 by L2 (Fig. 2E).

This simple pattern argues against the first model /Fig. 1D) in which multiple boundaries of mab-5 expression directly determine the pattern of cell fates. We do not see multiple boundaries of expression; instead, the spatial profile of mab-5 expression is similar in all of the P-cell descendants. The anterior boundary of expression, which lies between the P6 and P7 descendants, coincides with only one of the cell-fate decisions: that occurring in the Pn.p cells. To learn whether mab-5 expression actually determines the location of this boundary, we examined the effects of ectopic mab-5 expression using both a mab-5 gain-of-function promoter mutant, e1751(gf), and a heat shock promoter fusion.

mab-5 expression determines which Pn.p cells fuse with the epidermal syncytium

In Pn.p cells, mab-5 determines whether cells will remain mononucleate or fuse with the epidermal syncytium (Kenyon 1986). Pn.p cells 7-11 in the normal male are affected by mab-5 activity, whereas 1-6 are not (Fig. 1C). To alter the pattern of mab-5 expression, we first used the promoter mutation mab-5(e1751gf) described previously (Hedgecock et al. 1987; Salser and Kenyon 1992). In this background the domain of mab-5 expression extended anteriorly to include every P-cell descendant (Fig. 2G). Moreover, the fusion decisions of Pn.p cells 1-6 as well as 7-11 were affected (see below; Fig 3E-F). Thus, the boundary between affected and unaffected Pn.p cells appears to be determined by the boundary of mab-5 expression.

mab-5 interacts combinatorially with lin-39 in Pn.p cells

Remarkably, within the domain of mab-5 expression in the experiment just described, its effect on cell fusion depended on interactions with another HOM-C gene, lin-39. Previous work (Clark et al. 1993; Wang et al. 1993) has shown that when neither gene is active, $\mathrm{P}(1-$ 11).p cells all fuse with the epidermal syncytium (Fig. $3 \mathrm{~A})$. When either mab-5 or lin-39 acts on its own, it can prevent fusion of the Pn.p cells in its domain of activity (Fig. 3B,C). Finally, where the domains of mab-5 and 

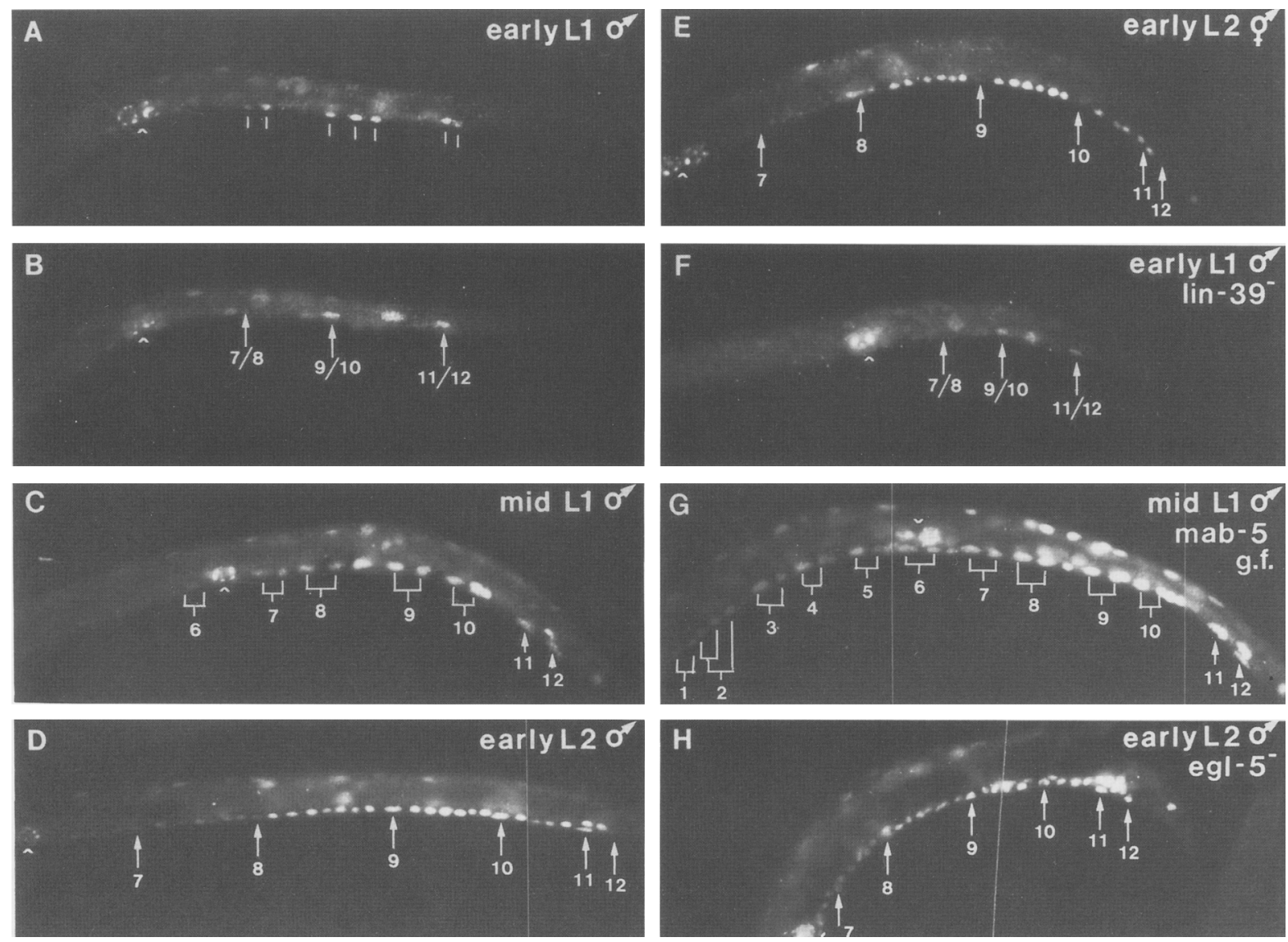

Figure 2. Mab-5 protein distribution in larvae. $(A-B)$ Early L1 male. On the right side $(B), \mathrm{P} 9 / 10$ and $\mathrm{P} 11 / 12$ stain, as do their bilaterally symmetric homologs on the left (not shown). The more anterior P1/2, P3/4, P5/6, and P7/8 cells are unstained. The epidermal cell V6, M myoblast, and several posterior body wall muscles also stain. In the ventral nerve cord $(A)$, seven embryonically produced juvenile motoneurons, DB6-DA8, stain. This pattern is reproducible $(n=61$ sides, $\geqslant 25$ male, $\geqslant 17$ hermaphrodite, $n=17$ ventral, 10 male, 7 hermaphrodite). Nonspecific germ-line staining is indicated ( $\wedge$ )(see Materials and methods). $(C)$ Older L1 male $(\sim 12$ hr after hatching). P1-P10 have divided; P11-P12 have not. Mab-5 is detected in P7-P12 or descendants but not in P1-P6 descendants. Juvenile motoneurons continue to stain $(n=14$ male, 3 hermaphrodite). $(D)$ Early L2 male $(n=38)$. Pn.p nuclei are indicated. Other $P$ descendants are mixed with juvenile motoneurons and cannot be distinguished unequivocally on the basis of morphology or staining. P12 descendants do not stain at this stage. $(E)$ Early L2 hermaphrodite. P(9-11).p cells have fused with the epidermal syncytium and no longer express mab-5 $(\uparrow) . \mathrm{P}(9-11)$.aap cells have undergone programmed cell death. Aside from these differences, expression is identical to that in the male $(n=18)$. $(F)$ lin-39(n1760) early Ll male. As in wild type, P7/8 does not stain at this stage $(n=16)$. Expression first appears when P7 $\sim 8$ cells enter the ventral cord $(n=6)$ and after they divide $(n=3)$. The pattern is indistinguishable from wild type, although fainter. $(G) \mathrm{mab}-5(\mathrm{e} 1751 \mathrm{gf}) \mathrm{Ll}$ male $\sim 12 \mathrm{hr}$ post hatching. All $\mathrm{P}$ descendants stain $(n=10)$. $(H)$ egl-5( $-\mid$ early L2 male. Mab-5 is not only detected in P9-P11 descendants, as in wild type $(D)$, but also in P12.p and in four additional cells that appear to be the P12.a descendants $(n=6)$. In hermaphrodites, only three additional cells stain, presumably because of the death of P12.aap in this background $(n=13)$. Precursors of these cells can be identified unambiguously and consistently exhibit Mab-5 labeling $(n=8)$.

lin-39 activity overlap in the wild-type male, something unexpected happens: lin-39 and mab-5 effectively neutralize one another, leading to fusion of Pn.p cells 7-8 (Fig. 3D).

Several models could account for this interaction. One possibility is that lin-39 and mab-5 simply repress each other transcriptionally in the region of overlap /cells 7-8). Thus, neither gene would achieve a high enough level of activity to prevent cell fusion. Consistent with the model, we did find that mab-5 expression was lower in the $P(7-8)$ lineages than in other $P$ lineages: As already described, it is undetectable in $\mathrm{P} 7 / 8$ at the time of hatch- ing and only barely detectable in the final $\mathrm{P}(7-8)$ descendants.

Further observations, however, argue against the mutual repression model. First, no increase in Mab-5 levels was seen in $\mathrm{P} 7 / 8$ or $\mathrm{P}(7-8) \cdot \mathrm{p}$ in a lin-39(-) background (Fig. 2F). Conversely, a lin-39-lacZ fusion was expressed strongly in both $\mathrm{P} 5 / 6(83 \%)$ and $\mathrm{P} 7 / 8(96 \%, n=24)$, and expression was not increased in a mab-5(-) background $(57 \%, 82 \%, n=28)$. Finally, according to the mutual repression model, the level of Mab-5 protein in the zone of overlap (where cells fuse) should be lower than the level outside this region (where cells remain unfused). In 

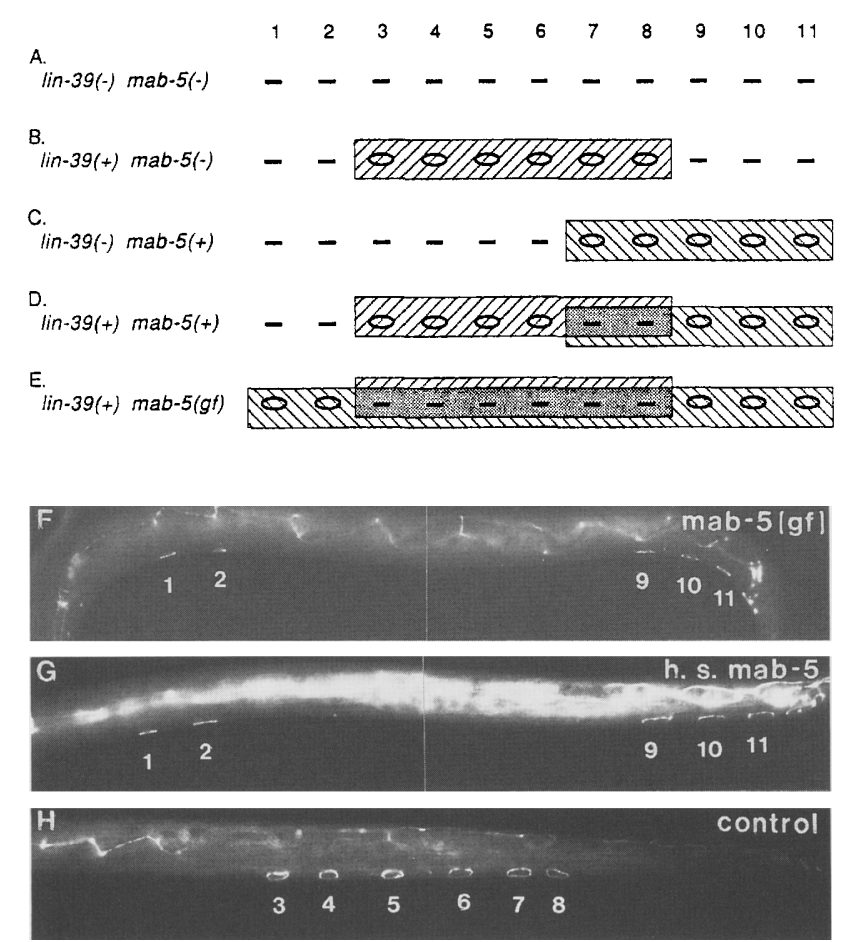

Figure 3. mab-5 and lin-39 interact combinatorially to influence the fusion of Pn.p cells. $(A-E)$ Schematic diagram of Pn.p cell fates in various mutant backgrounds. Ovals represent unfused Pn.p cells; dashes represent Pn.p cells that have fused with the surrounding syncytium. Domains of $m a b-5$ and lin-39 activity are hatched, and the overlap is darkly shaded. The indicated mab-5(gf) phenotype applies to both e1751gf and to $m a b-5|-|$ animals expressing $m a b-5$ under heat shock control. $(F)$ Representative pattern of fusion in mab-5(e1751gf) male visualized by staining with $\mathrm{mAb} M H 27$. The outlines of $\mathrm{P}(1-2$ and 9-11).p are still visible, whereas $\mathrm{P}(3-8)$.p cells have fused with the syncytium and are no longer detectable. In a population of e1751 males, the frequencies with which $\mathrm{P}(1-11) \cdot \mathrm{p}$ remained unfused were, respectively, $100 \%, 100 \%, 12 \%, 0 \%, 2 \%, 2 \%$, $0 \%, 0 \%, 100 \%, 100 \%$, and $100 \%(n=52) .(G)$ Fusion pattern in a mab-5 $|-|$ male expressing $m a b-5$ under heat shock control. The outlines of $\mathrm{P}(1-2$ and $9-11) . \mathrm{p}$ remain visible, whereas $\mathrm{P}(3-$ 8).p cells have fused with the syncytium. The frequencies with which $\mathrm{P}(1-11) \cdot \mathrm{p}$ cells remained unfused were, respectively, $45 \%, 58 \%, 27 \%, 3 \%, 12 \%, 6 \%, 9 \%, 3 \%, 91 \%, 78 \%$, and $87 \%$ $(n=32)$. For comparison, see control results below. Hermaphrodites were relatively unaffected: The frequencies with which hermaphrodite Pn.p cells remained unfused were, respectively, $2 \%, 5 \%, 93 \%, 98 \%, 100 \%, 100 \%, 100 \%, 98 \%, 15 \%, 20 \%$, and $32 \%(n=60) .(H)$ Representative mab-5 $|-|$ control male that was heat-shocked in the same manner as that shown in $G$ but does not carry the heat shock promoter-mab-5 fusion construct. $\mathrm{P}(3-8) . \mathrm{p}$ always remain unfused, whereas $\mathrm{P}(1-2$ and $9-11)$.p always fuse $(n=25)$ animals. Similarly, in animals carrying the construct but not exposed to high temperatures, $\mathrm{P} / 3$ 8).p cells almost always remain unfused and $\mathrm{P}(1-2$ and 9-11).p cells almost always fuse. The frequencies with which $\mathrm{P}(1-11) \cdot \mathrm{p}$ remain unfused are: $0,0,100 \%, 98 \%, 100 \%, 100 \%, 100 \%$, $100 \%, 2 \%, 4 \%, 4 \%(n=53)$.

mab-5(1751gf) larvae, however, mab-5 was expressed at comparable levels in P2.p and P3.p (Fig. 2G) despite the fact that it has opposite effects on these two cells. In
P2.p, mab-5(e1751gf) prevents fusion, whereas in P3.p, mab-5(e1751gf) induces fusion (Fig. 3E-F).

These observations suggest that the antagonism between mab-5 and lin-39 does not occur at the level of transcription. To eliminate this possibility more convincingly, we expressed mab-5 under the control of a heterologous promoter immune to the effects of lin-39. We placed Mab-5 under heat shock control in a mab5(-) background. In control animals (not heat-shocked or lacking the fusion construct), the pattern of fates was identical to that seen in mab-5 - ) larvae (Fig. $3 \mathrm{H}$ ). In males induced to express mab-5 ubiquitously, however, the pattern was completely inverted (Fig. 3G). Pn.p cells in the lin-39 domain (3-8) fused with the syncytium, whereas those outside this domain (1-2 and 9-11) remained unfused. Because a heterologous promoter was used to drive mab-5 expression in these experiments, transcriptional repression of $m a b-5$ by $l i n-39$ is not required for the two genes to neutralize one another. Instead, these data and the observed expression patterns argue that post-translational interactions cause effective cancellation where both genes are expressed.

The effects of mab-5 on cell fusion are known to be specific to the male (Kenyon 1986). Because mab-5 expression is observed in both male and hermaphrodite Pn.p cells, mab-5 must somehow be prevented from functioning in the hermaphrodite. Consistent with this, we found that ectopic mab-5 expression under heat shock control, or in e1751 $\mathrm{gf}$ larvae, had little or no effect on the hermaphrodite Pn.p fusion pattern [see legend to Fig. 3(G)]. mab-5 expression both failed to prevent fusion of Pn.p cells outside the lin-39 domain and failed to antagonize lin-39 in the region of overlap. Thus, although HOM-C gene expression along the $\mathrm{A} / \mathrm{P}$ axis appears to be regulated similarly in males and hermaphrodites, the downstream effects on cell fates can be modulated. In $C$. elegans, sex determination has been shown to proceed through a cascade of gene interactions culminating in hermaphrodite-specific expression of the zinc finger transcription factor tra-1 (Zarkower and Hodgkin 1992). In hermaphrodites, where tra-1 is active, mab-5 has no effect. One possibility is that tra-1 interferes directly with the binding or activity of $m a b-5$ on the promoters of target genes.

\section{lin-39 activity, not mab-5 expression, positions the boundary between cell fates in Pn.aap cells}

In Pn.aap cells of the male, mab-5 controls a different decision: whether to produce a single neuron or to divide again to produce two neurons. mab-5 is required for Pn.aap cells 9-11 to divide (Fig. 4A), and without it they behave like P2.aap cells, becoming single neurons with uniquely positioned nuclei (Fig. 4B; Kenyon 1986). When we expressed mab-5 ectopically using the promoter mutation $e 1751 g f$, Pn.aap cells 1 and 2 divided consistently, showing that mab-5 activity is sufficient to produce this division (Fig. 4C). In the wild type, then, mab-5 activity must be off in cells $1-2$ and on in 9-11, consistent with the expression pattern that we have seen. 


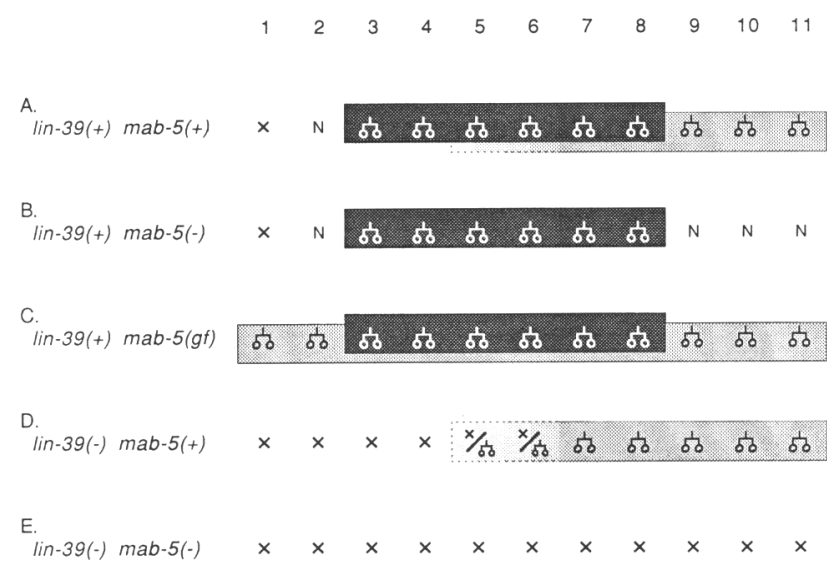

Figure 4. lin-39 obscures the underlying boundary of mab-5 activity in Pn.aap cells. $(A-E)$ Schematic diagram of Pn.aap cell fates in various mutant backgrounds. $(x)$ Programmed cell death; $(\mathrm{N})$ single neurons with compact nuclei positioned dorsally in the cord. Pairs of motoneurons are represented with white circles; the posterior of each pair contains the neurotransmitter serotonin (Loer and Kenyon 1993). Pairs of nonserotonergic cells are represented with black circles; EM reconstructions of wild type suggest that one of each pair is an interneuron, the other makes no known electrical or chemical junctions (J. White, pers. comm.). Domains of mab-5 activity are shaded; lin-39 activity in promoting production of motoneurons is shown in black. lin-39 is also active outside this domain in preventing cell death. $(C)$ Four mab-5(e1751gf) lineages were followed in males. $\mathrm{P}(1-12)$.aap cells always divided. Analysis of serotonin immunoreactivity demonstrates that only the descendants of $\mathrm{P}(3-8)$. aap cells express serotonin $(n=97) .\{D\rangle$ Four lineages were followed in males: one in a lin-39(mu26) background, and three in a lin-39(n1760) background. In all four lineages, $\mathrm{P}(1-4)$.aap cells underwent programmed cell death, whereas $\mathrm{P}(7-11)$.aap cells survived. The fate of $\mathrm{P}(5-6)$.aap cells was variable: in mu26 they both died, in one $n 1760$ lineage, both died, in another, only P5.aap died, and in the third, neither cell died. Surviving cells generally went on to divide in L3, the only exceptions being a single P7.aap cell in mu26, and a single P8.aap cell in $n 1760$. Although these cells swelled up during L3, they never divided. $(E)$ A single lineage was followed in a lin39(n1760) mab-5(e1239) male. P(1-11).aap all underwent programmed cell death. P12.aap survived, presumably because of egl-5 activity.

Pn.aap cells 3-8, in the central body of the male, adopt a different fate: They divide to produce two motoneurons, one of which contains the neurotransmitter serotonin (Loer and Kenyon 1993). None of the neurons produced by cells $9-11$ is a motoneuron or contains serotonin. Loss of mab-5 $(n=44)$, egl-5 $(n=36)$, or both activities $(n=39)$ did not affect the production of these serotonergic neurons. Even in mab-5(e1751gf) larvae, $\mathrm{P}(3-8)$.aap consistently divided and produced six serotonergic neurons ( $n=97$; Fig. $4 \mathrm{C}$ ). We wanted to know why Pn.aap cells in the middle of the body adopt this different fate and why they fail to respond to mab-5.

Previous work has shown that the HOM-C gene lin-39 is required for production of these serotonergic motoneurons in the mid-body region (Loer and Kenyon 1993). To see whether lin-39 might obscure the underlying activity of $m a b-5$ in $\mathrm{P}(3-8)$.aap cells, we examined the pattern of cell divisions in a lin-39|-) background. Indeed, in lin39| - | larvae a new functional boundary was seen, which coincided with the border of mab-5 expression: Posterior Pn.aap cells 7-11 divided to produce nonserotonergic neurons, whereas cells 1-6 underwent programmed cell death (Fig. 4D). The boundary was somewhat variable: Often P6.aap cells, and sometimes even P5.aap cells, divided like 7-11. To see whether these cell divisions reflect mab-5 activity, we examined the pattern of cell fates in a lin-39 mab-5 double mutant. In these animals, cells 1-11 all did undergo programmed cell death (Fig. $4 \mathrm{E})$, showing that mab-5 activity is required for the survival and division of Pn.aap cells 7-11 (and sometimes 5 and 6) in a lin-39|- | background. This spatial pattern of $m a b-5$ activity corresponds with the observed expression pattern of mab-5 and the pattern of activity seen in Pn.p cells. In the wild-type, however, lin-39 activity overlaps with the domain of mab-5 expression and predominates, causing cells $7-8$ to produce motoneurons.

Both mab-5 and lin-39, then, suffice to promote the division of Pn.aap cells, but they specify different roles and neurotransmitter phenotypes for the resulting neurons. Unexpectedly, lin-39 not only was required for division of Pn.aap cells 3-8 to produce motoneurons but also for survival of $\mathrm{P} 2$.aap cells in the wild-type and cells $9-11$ in a mab-5|-| background. We do not yet understand why lin-39 activity promotes the division of cells in the 3-8 region but merely prevents the death of cells 2 and 9-11. The lin-39-lacZ fusion was expressed at detectable levels in $\mathrm{P}(3-8)$ lineages but not outside this domain (data not shown; see also Wang et al. 1993). It therefore seems likely that the level or timing of lin-39 expression is important in creating these differences in the final effect, although we cannot rule out the involvement of other regionally expressed genes.

\section{mab-5 is necessary but not sufficient to promote} cell death in Pn.aaap cells

In Pn.aaap cells 11-12, mab-5 is required to specify programmed cell death (Fig. 5A). Without mab-5, these cells instead appear to adopt the fate of cells 1-10, which are VB motoneurons (Kenyon 1986; Fig. 5B). In sharp contrast to this pattern of fates, high levels of mab-5 expression were observed in cells 9-10 in the wild type, which do not undergo programmed cell death (Fig. 2D). This suggested that Pn.aaap cells 11-12 might be the only homologs competent to respond to mab-5 in this manner. To test this, we expressed Mab- 5 using the heat shock construct in a mab-5|-| background. Only cells 11 and 12 responded consistently by undergoing programmed cell death (Fig. 5C). From this, we infer the presence of an unidentified gene activity in the posterior that cooperates with mab-5 or, conversely, some activity in the anterior that antagonizes mab-5. We have ruled out the involvement of other known HOM-C members; neither lin-39 nor egl-5 mutations affected the pattern of VB cell deaths $(n=22, n=11$, respectively). 


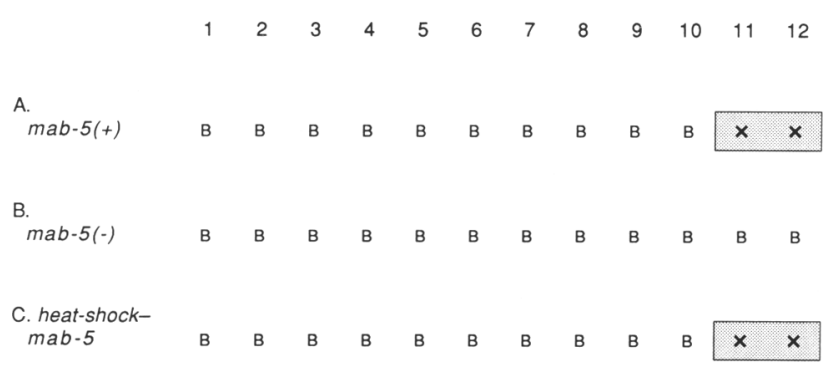

Figure 5. mab-5 is necessary but not sufficient to induce programmed cell death of Pn.aaap cells. $(A-C)$ Diagram of Pn.aaap fates. $(\times)$ Programmed cell death; (B) VB motoneurons. Shading represents $m a b-5$ activity rather than expression. $(C)$ Four lineages were performed in mab-5|-| males expressing mab-5 under heat shock control. In 4/4 animals P11.aaap cells underwent programmed cell death; in 1/4 animals, P12.aaap cells died; $\mathrm{P}(6-10)$.aaap cells always survived; no cell deaths were observed anterior to P6.aaap. A time course was done in a ced-1(-);mab$5(-)$ background, to determine the best time to induce mab-5 expression (see Materials and methods). The optimal time to elicit death of $\mathrm{P}(11-12)$.aaap cells was broad: $8-14 \mathrm{hr}$ post-hatching. P12.aaap deaths were observed in $50 \%$, P11.aaap deaths in $27 \%$, and rarely were there deaths in the position of P10.aaap $(3 \%, n=70)$. No more anterior cell deaths were seen.

mab-5 expression in $P 12$ descendants is repressed by egl- 5

Although Mab-5 was detected at high levels in P12 itself, expression declined during the P12 divisions and was not observed in the final descendants (Fig. 2, cf. B and D). Previous work has shown that mutations in the more posterior HOM-C gene egl-5 cause homeotic transformations in which P12 adopts a P11-like fate. This suggested that in egl-5|-| larvae, mab-5 might be expressed in descendants of both P11 and P12. In egl-5(-) larvae, we found strong mab-5 expression in P12 descendants at all stages of division, including the final pattern in the second larval stage (Fig. $2 \mathrm{H}$ ). We infer that in the wild type, eg1-5 activity leads to the repression of mab-5 expression in the P12 lineage.

We were surprised to find that mab-5 expression disappears in the P12 lineage long before the P12.aaap cell is born. This cell requires mab-5 activity to undergo programmed cell death. To resolve the question of when the Pn.aaap fate is specified, we carried out a time course using the heat shock-mab-5 construct to induce $m a b-5$ expression at different times in development (see legend to Fig. 5). We found that the most effective time to induce mab-5 expression to specify Pn.aaap cell death is between 8 and $14 \mathrm{hr}$ post-hatching, 2-8 hr before the birth of these cells. Thus, specification of the cell death appears to occur before the cell is born, but after the division of the $\mathrm{P}$ cells, consistent with the observed mab-5 expression pattern in the P12 lineage.

\section{Discussion}

In the ventral nerve cord of $C$. elegans, the stereotyped divisions of 12 precursor cells lead to the production of a repeating pattern of neuronal cell types. Position-specific variation in the fates of these repeated cell types is established through the localized activity of the HOM-C genes, much as segmental diversity is produced in Drosophila. The reproducible lineage and fate of these cells has provided us with an opportunity to correlate patterns of HOM-C gene expression and function at the level of single cells. We have examined the role of one HOM-C gene, mab-5, in patterning the posterior ventral nerve cord. We find that interactions between this gene and other HOM-C members play a significant role in establishing the final pattern of cell fates.

mab-5 affects the fates of three types of P-cell descendants in the male: the Pn.p cell, which is epidermal, the Pn.aap cell, which is a sex-specific neuronal cell, and the Pn.aaap cell, which is a type-VB motoneuron. Each of these cell types is repeated 12 times along the length of the body; and, in each case, mab-5 causes a contiguous group of cells in the posterior to adopt specialized fates. However, the boundary between specialized fates and more anterior fates occurs in a different $\mathrm{A} / \mathrm{P}$ location for each of these decisions. We have found that mab-5 activity is regulated in three different ways to produce these boundaries (Fig. 6A).

In Pn.p cells, the level of Mab-5 protein itself serves to position a boundary between cells that fuse with the syncytium, and those that remain mononucleate. Mab-5 is expressed in a simple contiguous block of cells in the posterior nerve cord. The anterior boundary of this expression, between P6.p and P7.p, coincides with a boundary between fused and unfused cells. When the domain of expression is expanded anteriorly, the domain of mab-5 function also expands.

In contrast, in Pn.aap cells, lin-39 activity, rather than mab-5 expression, produces the boundary between different cell fates. Previous work has shown that lin-39 promotes the production of motoneurons by $\mathrm{P}(3-8)$.aap. We find that in these cells lin-39 activity is dominant over $m a b-5$ and that this obscures the underlying pattern of mab-5 activity. In the absence of lin-39, an underlying border is revealed: $m a b-5$ activity extends to $\mathrm{P}(7-8)$.aap, in agreement with the observed expression pattern and pattern of activity in Pn.p cells. Analogous interactions occur between the corresponding genes in Drosophila: Mutations in Scr lead to transformations in segmental identity that reveal the underlying activity of Antp (Struhl 1982; Kaufman et al. 1990).

In Pn.aaap cells too, the boundary between cell fates does not result from differences in mab-5 expression. Mab-5 is not only expressed strongly in P11.aaap cells, where it induces programmed cell death, but also in P10.aaap, which becomes a VB motoneuron. Moreover, if Mab-5 is expressed ubiquitously, under the control of a heat shock promoter, only $\mathrm{P}(11-12)$.aaap cells respond efficiently. This suggests the presence of a cooperative gene activity in the posterior, or alternatively, an antagonistic activity in the anterior. Other known HOM-C members (lin-39 and egl-5) do not affect the decision.

Not only do the three distinct anterior boundaries of mab-5 activity arise from the localized action of three 
A

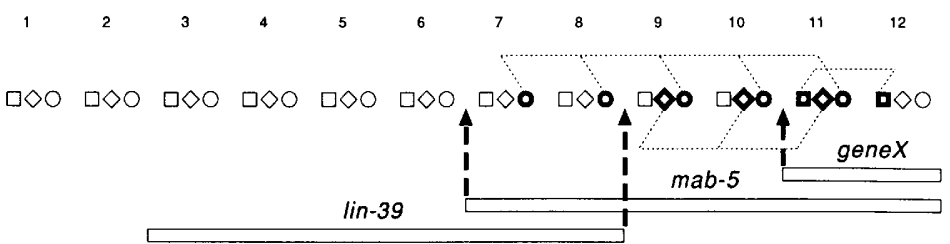

B

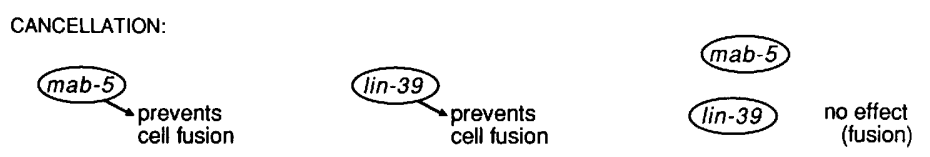

DOMINANCE:

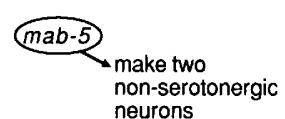

neurons
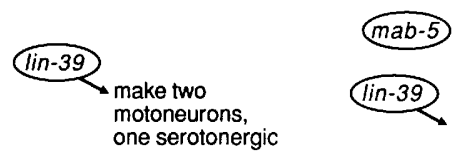

(iin-39) make two

motoneurons,

COOPERATION:
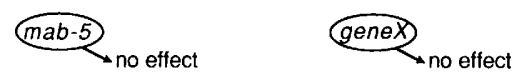

Figure 6. Genes that regulate the pattern of cell fates in the posterior nerve cord. $(A)$ Localized activities of three different genes position boundaries in cell fate. In Pn.p cells (circles), the pattern of responding cells is determined by mab-5 expression. In Pn.aap cells (diamonds), overlapping lin-39 activity obscures effects of mab-5 in $\mathrm{P}(7-8)$.aap. In Pn.aaap cells (squares), mab-5 can induce only $\mathrm{P}(11-12)$ aaap to die. This may reflect localized activity of a coactivator in the posterior (as shown) or an antagonistic activity in the anterior. $(B)$ Interactions between these genes vary depending on the cell type. In Pn.p cells, mab-5 and lin-39 each prevent cell fusion; but where both are expressed, they effectively cancel. In Pn.aap cells, lin-39 is dominant over mab-5 and promotes motoneuron production. In Pn.aaap cells, mab-5 and a postulated gene activity may cooperate to induce programmed cell death. different genes, but where these gene activities overlap, they interact in different ways depending on the cell type (Fig. 6B). They can neutralize one another, one gene can predominate over the other, and mab-5 may cooperate with an unidentified gene in the posterior.

In Pn.p cells, both lin-39 and mab-5 prevent cell fusion (see Fig. 6B); however, they effectively cancel each other out with respect to this decision in the zone of overlap. We have shown that this cancellation does not result from mutual transcriptional repression. Instead, whenever both genes are expressed, they antagonize one another's activity. This occurs whether Mab-5 is expressed under its own promoter, a rearranged promoter with ectopic activity, or a heat shock promoter. The ratio of mab-5 to lin-39 does not seem to be crucial for cancellation, as each of these methods of expression leads to somewhat different levels of Mab-5. Therefore, it seems unlikely that the two proteins cancel through formation of inactive heterodimers; instead, they may interact on the promoters of downstream genes involved in cell fusion. For example, mab-5 and lin-39 might each serve to repress a gene involved in cell fusion; but in combination, the genes could either fail to repress or might even activate expression of the fusion program.

In Pn.aap cells, lin-39 and mab-5 do not cancel each other out; instead lin-39 overrides mab-5 function. Thus, the same pair of genes can interact differently depending on the cell type or the decision that they are controlling. Previous work in Drosophila, although controversial, has suggested the presence of a functional hierarchy between HOM-C genes, termed posterior prevalence (González-Reyes et al. 1990). Our work suggests that prevalence of one gene over another is not necessar- ily a property of the gene products themselves but, instead, reflects the type of decision they are controlling and/or the cellular environment.

In Pn.aaap cells, mab-5 appears to interact with an unidentified gene activity outside the HOM-C. $A b d-B$ function in Drosophila appears to be similarly regulated in parasegments 14 and 15 by the localized activity of the spalt gene, which is also not a member of the HOM-C (Jürgens 1987,1988).

These three interactions are sufficient to explain the staggered anterior border of mab-5 activity. In the posterior, we have found that the HOM-C gene egl-5 represses mab-5 expression in the P12 lineage. Similar interaction is seen at the transcriptional level between the corresponding Drosophila genes $U b x$ and $A b d-B$ (Struhl and White 1985; Casanova et al. 1986; Casanova and White 1987; Karch et al. 1990; Macías et al. 1990). Although the functional significance of this interaction has been questioned previously (González-Reyes et al. 1990), more recent work suggests that it plays a significant role in restricting $U b x$ activity (Lamka 1992). It may be that in C. elegans, repression of mab-5 by egl-5 helps to limit its domain of activity.

Previous analysis of the mab-5 phenotype suggested that the P2 lineage might be representative of the ground state, that is, the underlying pattern that is modified through the action of the HOM-C genes (Kenyon 1986). In mab-5 mutants, cells P9-P12 all do adopt a P2-like fate. Our further analysis of the lin-39 mab-5 double mutant, however, suggests that the ground state may instead be P1-like. In the double mutant background, P(211).aap cells all undergo programmed cell death like P1.aap. Moreover, the other $\mathrm{P}(2-11)$ descendants behave 
similarly to Pl descendants as far as their pattern of division and/or fusion. Complete resolution of the issue of the ground state may depend on the isolation of mutations in the C. elegans labial homolog ceh-13.

Currently, it is not known what mechanism generates the graded pattern of mab-5 expression seen in both the $P$ descendants and juvenile motoneurons. One possibility is that similar, perhaps positional, mechanisms regulate mab-5 expression levels in the two cell types. Certain aspects of the mab-5 expression pattern imply that cell-extrinsic mechanisms are used to regulate the level of Mab-5. The P9/10 cells arise from lineally identical positions on the left and right sides of the animal and interdigitate at random when they move into the ventral cord (Sulston and Horvitz 1976). When they first enter the cord they express apparently equal levels of Mab-5. Nevertheless, P10 descendants consistently come to express higher levels of Mab-5 than P9 descendants. This may reflect signaling between the $\mathrm{P}$ cells themselves or other positional cues.

Interestingly, we see mab-5 expression not only in the posterior motoneurons, where its function is unknown, but also in the posterior body muscles. This raises the possibility that HOM-C genes play a role in neuromuscular junction specificity. We also find, however, that these genes may play a more basic role in muscle attachment. Mutations in both mab-5 and lin-39 lead to defects in ventral body-wall muscle attachment in their domains of expression (see Materials and methods).

In summary, we have examined the role of one HOM-C gene in patterning the posterior ventral nerve cord. We find that diverse interactions between this gene and other HOM-C members play a significant role in regulating the final pattern of cell fates. Both cross-regulation of expression and several types of post-translational interactions are important. Some of the interactions that we have observed have been described previously in Drosophila at the level of whole body segments. We find that these interactions can also occur at the level of single cells and that they vary depending on the cell type. The flexibility of these interactions suggests that overlapping domains of HOM-C expression serve simply as raw material for patterning cell fates: The basic information can be logically integrated and interpreted in many different ways, depending on the cell type and the requirements of the organism.

\section{Materials and methods}

Nematode strains

Nematode strains were maintained as in Brenner (1974) and Wood et al. (1988). Analysis was done at $20^{\circ} \mathrm{C}$ except for heat shock inductions. Males were generated using him-5(e1490), which boosts incidence of male self-progeny. Other mutations used were mab-5(e2088) and mab-5(e1239), which do not express detectable levels of Mab-5 (see below) and appear to be null by genetic criteria (Kenyon 1986); mab-5(e1751gf) (Hedgecock et al. 1987), a large tandem duplication of the gene that alters promoter activity (Salser and Kenyon 1992) leading to increased and ectopic expression; lin-39(n1760) (Clark et al.
1993) and lin-39(mu26) (Wang et al. 1993), both strong alleles, one of which (mu26) disrupts the homeo domain; egl-5(n945), an amber mutation that appears null by genetic criteria (Chisholm 1991); and ced-1(e1735), which prevents engulfment of dying cells (Hedgecock et al. 1983).

\section{Antiserum preparation}

mab-5-coding sequences were cloned in Escherichia coli vectors pGEX-3X and pAR3040 to produce Mab-5-glutathione- $S$ transferase (GST) and Mab-5- $\beta$-galactosidase ( $\beta$-gal) fusions. Both were insoluble and purified from inclusion bodies by SDSPAGE. Electroeluted proteins were administered to six rabbits. Immunizations were $250 \mu \mathrm{g}$ in complete Freund's adjuvant, followed by $150-\mu \mathrm{g}$ boosts in incomplete Freund's adjuvant at 4 -week intervals. A rabbit immunized with $\beta$-gal fusion and boosted with GST fusion gave the best response. Sera were tested on larvae expressing Mab-5 under heat shock control. Preimmune sera did not react in this assay.

Following the fourth boost, sera were affinity purified against Mab-5-GST on nitrocellulose and eluted in $0.2 \mathrm{M} \mathrm{NaCl} / 0.2 \mathrm{M}$ glycine (pH 2.5). Greater than $80 \%$ of reactivity was recovered. This preparation cross-reacts with germ-line granules, a common problem with rabbit and mouse sera. Germ-line reactivity is not attributable to Mab-5, as it is present in mab-5 - | strains. Nuclear staining appears specific, as it is absent in mab5(e2088) and mab-5(e1239). Before use, affinity-purified antibodies were preadsorbed to $m a b-5(e 2088)$ larvae to reduce background. Larvae were prepared as described below but equilibrated in $5 \mathrm{~mm}$ EDTA/ $5 \mathrm{~mm}$ EGTA/0.01\% $\mathrm{NaN}_{3} / \mathrm{TBSTw}$ (137 $\mathrm{mm} \mathrm{NaCl}, 2.7 \mathrm{~mm} \mathrm{KCl}, 25 \mathrm{~mm}$ Tris at $\mathrm{pH} 7.7,0.5 \%$ Tween 20 ). Sixty-five microliters of sonicated larvae was blocked with $1 \%$ BSA and $5 \%$ goat serum in $90 \mu \mathrm{l}$. Ten microliters of purified antibody was added and preadsorbed overnight at $37^{\circ} \mathrm{C}$. Debris was removed with a 30 -min microcentrifuge spin.

\section{Antibody staining of whole-mount larvae}

For Mab-5 staining, larvae were prepared as described (Finney and Ruvkun 1990), with changes: 10-100 $\mu$ l of larvae was frozen in $1 \mathrm{ml}$ of ice-cold $1 \%$ paraformaldehyde in $\mathrm{MRWB} 180 \mathrm{mM} \mathrm{KCl}$, $20 \mathrm{~mm} \mathrm{NaCl}, 10 \mathrm{~mm}$ EGTA, $5 \mathrm{~mm}$ spermidine $\mathrm{HCl}, 15 \mathrm{~mm} \mathrm{Na}$ PIPES at $\mathrm{pH} 7.4,25 \% \mathrm{MeOH})$. Tubes were thawed and fixed overnight at $4^{\circ} \mathrm{C}$. Subsequent washes and incubations were $1 \mathrm{ml}$ at $25^{\circ} \mathrm{C}$ unless indicated. Larvae were rinsed twice in ice-cold Tris-Triton (100 mM Tris at $\mathrm{pH} 7.4,1 \%$ Triton X-100, $1 \mathrm{~mm}$ EDTA), incubated for $2 \mathrm{hr}$ at $37^{\circ} \mathrm{C}$ in $2 \% \mathrm{BME} /$ Tris-Triton, rinsed in borate ( $25 \mathrm{mM} \mathrm{NaBO}_{3}$ at $\mathrm{pH} 9.2$ ), incubated for $15 \mathrm{~min}$ in $30 \mathrm{mM} \mathrm{DTT/borate,} \mathrm{rinsed} \mathrm{twice} \mathrm{in} \mathrm{borate,} \mathrm{incubated} \mathrm{for} 15$ min in $1 \% \mathrm{H}_{2} \mathrm{O}_{2}$ /borate, and rinsed again in borate. Steps in borate were repeated to further permeabilize larvae. The larvae were then rinsed twice in $0.001 \%$ Triton X-100 and settled onto polylysine slides, $22-\mathrm{mm}^{2}$ coverslips were applied, excess liquid was removed, slides were frozen on dry ice, and coverslips were pryed off. Slides were thawed in $30 \% \mathrm{EtOH}$ in TBSTw, rinsed in TBSTw, blocked in $1 \% \mathrm{BSA} / 5 \%$ goat serum/TBSTw for $30 \mathrm{~min}$ at $37^{\circ} \mathrm{C}$, incubated with $15 \mu \mathrm{l}$ of preadsorbed primary antibody for $2 \mathrm{hr}$ at $37^{\circ} \mathrm{C}$, rinsed $4 \times 1 \mathrm{hr}$ in $37^{\circ} \mathrm{C}$ TBSTw, reblocked, incubated for $2 \mathrm{hr}$ with rhodamine goat anti-rabbit $(1: 200)$ in $1 \% \mathrm{BSA} / 5 \%$ goat serum/ TBSTw, and rinsed $3 \times 15 \mathrm{~min}$ at $37^{\circ} \mathrm{C}$. Larvae were mounted in $80 \%$ glycerol, $2 \% n$-propyl gallate, and $5 \mu \mathrm{g} / \mathrm{ml}$ of DAPI and photographed with Kodak Tech Pan. Costaining with anti-histone monoclonal antibody (Chemicon MAB052) was used to gauge tissue accessibility.

Pn.p cell fusion was visualized as described (Kenyon 1986), using the mouse mAb MH27, which stains a component of 
septate junctions (Francis and Waterston 1991). Serotonin immunoreactivity was detected as described (Desai et al. 1988), with slight modifications (Loer and Kenyon 1993).

\section{Expression of Mab-5 under heat shock control}

Integrants of the extrachromosomal array muEx14 (Salser and Kenyon 1992) were obtained by $\gamma$-irradiation (C. Kari, A. Fire, and R. Herman, pers. comm.). The array carries the marker unc$31(+)$ as well as the fusion placing mab-5 under the control of the hsp16.1 heat shock promoter (Stringham et al. 1992). Two of the integrants, muIs 9 and muIs10, gave reliable induction of $m a b-5$. These were outcrossed three times to unc-31(e169); him-5(e1490) to remove $\gamma$-induced mutations, and crossed into an mab-5(e2088); unc-31(e169); him-5(e1490) background. Both insertions are $\mathrm{X}$-linked, one close to lon-2.

To affect Pn.p fusion, larvae were given $31^{\circ} \mathrm{C}$ pulses, $15-30$ min in length, with 90 -min recovery periods at $20^{\circ} \mathrm{C}$. Pulses started 6-11 hr after hatching and continued for $12 \mathrm{hr}$, at which time larvae were prepared for MH27 staining. (Pn.p cells are born at $8-10 \mathrm{hr}$.) e2088; e169; e1490; muIs9 and e2088; e169; e1490; muIs10 gave similar results, and data were pooled. $e 2088$; $e 1490$ larvae given heat shocks in parallel were unaffected. e2088; e169; e1490; muIs 9 and e2088; e169; e1490; muIs 10 larvae grown at $20^{\circ} \mathrm{C}$ were also unaffected.

To determine the optimal time for $m a b-5$ induction to specify programmed cell death in Pn.aaap cells, e1735; e2088; e169; e1490; muIs9 larvae were given 30-min heat shocks at 6-8, $8-10,10-12,12-14$, or 14-16 hr post-hatch. Cell deaths were scored at $17-19 \mathrm{hr}$. Frequencies of cell death during these periods were $3 / 30,11 / 22,11 / 28,13 / 20$, and $3 / 20$ (for P12.aaap), $2 / 30,4 / 22,11 / 28,4 / 20$, and $4 / 20$ (for P11.aaap), and 0/30, 2/22, $0 / 28,0 / 20$, and $0 / 20$ (for P10.aaap). Deaths were identified on the basis of position and scored in males to avoid confusion with Pn.aap deaths. To definitively identify dying cells, four lineages were performed in e2088; e169; e1490; muIs 9 males. Fifteen-minute heat shocks were given at 2 -hr intervals starting after the division of P12.a ( $\sim 11$ hr post-hatch).

\section{Analysis of mutant lineages}

Larvae were mounted as described (Wood et al. 1988) and observed using Nomarski optics. Pn.aap divisions in $e 1751$ were observed to the end of L3. n1760, mu26, and n1760 e1239 L1 lineages leading to Pn.aap cells were followed. Occasional polarity reversals in P-cell divisions were observed in $n 1760$ and mu26. Cell deaths were noted, and larvae were allowed to develop on petri plates until $\mathrm{L} 3$. After remounting, divisions of surviving Pn.aap cells were observed to the end of L3. Detached ventral body-wall muscles were seen in mab-5 (8/26 e2088 late Lls between V5.a and anus), lin-39 (12/25 n1760 late L1s between V1.a and V4.p), as well as the double mutant (from V2.p back to the anus). Defects in P-descendant migrations were also seen: P1 descendants in wild type migrate anteriorly. In lin-39 larvae, $\mathrm{P}(2-5)$.a descendants migrated anteriorly like P1.a descendants in wild type. In lin-39 mab-5, $\mathrm{P}(1-11)$.a descendants migrated anteriorly. Finally, mab-5(1751gf) blocked anterior migrations of Pl.p and Pl.a.

\section{Acknowledgments}

We thank Mike Finney and Cori Bargmann for support and technical advice on Mab-5 immunostaining, David Drechsel, Tim Mitchison, Kenneth Sawin, Roland Bainton, and Ray Deshaies for advice on fusion protein preparation and antibody purifica- tion, Jason Swedlow for recommending MAB052 as a control, Bruce Wang and Naomi Robinson for the lin-39-LacZ fusion, Sue Parmelee for photographic assistance, Bill McGinnis, Matt Scott, Craig Hunter, Deborah Cowing, Judith Austin, Michael Müller-Immergluck, and other Kenyon laboratory members for discussions of the manuscript. Some nematode strains used in this work were provided by the Caenorhabditis Genetics Center, which is funded by the National Institutes of Health (NIH) National Center for Research Resources (NCRR). Other strains were kindly provided by Ed Hedgecock, Scott Clark, and Andrew Chisholm. This work was supported by an NIH grant to C.K., who is a Packard Foundation fellow. S.J.S. was supported by the National Science Foundation and Chancellor's predoctoral fellowships, and an NIH training grant. C.M.L. was supported by an NIH postdoctoral fellowship.

The publication costs of this article were defrayed in part by payment of page charges. This article must therefore be hereby marked "advertisement" in accordance with 18 USC section 1734 solely to indicate this fact.

\section{References}

Beachy, P.A., S.L. Helfand, and D.S. Hogness. 1985. Segmental distribution of bithorax complex proteins during Drosophila development. Nature 313: 545-551.

Brenner, S. 1974. The genetics of C. elegans. Genetics 77: 7194.

Bürglin, T.R., G. Ruvkun, A. Coulson, N.C. Hawkins, J. McGhee, D. Schaller, C. Wittmann, F. Müller, and R.H. Waterston. 1991. Nematode homeobox cluster. Nature 351: 703.

Carroll, S.B., M.A. Laymon, M.A. McCutcheon, P.D. Riley, and M.P. Scott. 1986. The localization and regulation of Antennapedia expression in Drosophila embryos. Cell 47: 113122.

Carroll, S.B., S. DiNardo, P. O'Farrell, R.A.H. White, and M.P. Scott. 1988. Temporal and spatial relationships between segmentation and homeotic gene expression in Drosophila embryos: distribution of the fushi-tarazu, engrailed, Sex combs reduced, Antennapedia, and Ultrabithorax proteins. Genes \& Dev. 2: 350-360.

Casanova, J. and R.A.H. White. 1987. Trans-regulatory functions in the $A b d o m i n a l-B$ gene of the bithorax complex. Development 101: 117-122.

Casanova, J., E. Sánchez-Herrero, and G. Morata. 1986. Identification and characterization of a parasegment specific regulatory element of the Abdominal-B gene of Drosophila. Cell 47: 627-636.

Celniker, S.E., S. Sharma, D.J. Keelan, and E.B. Lewis. 1990. The molecular genetics of the bithorax complex of Drosophila: Cis-regulation of the Abdominal-B domain. EMBO I. 9: 4277-4286.

Chisholm, A. 1991. Control of cell fate in the tail region of $C$. elegans by the gene egl-5. Development 111: 921-932.

Clark, S., A. Chisholm, and H.R. Horvitz. 1993. Control of cell fates in the central body region of $C$. elegans by the homeobox gene lin-39. Cell 74: 43-55.

Costa, M., M. Weir, A. Coulson, J. Sulston, and C. Kenyon. 1988. Posterior pattern formation in C. elegans involves position-specific expression of a gene containing a homeobox. Cell 55: 747-756.

Desai, C., G. Garriga, S.L. McIntire, and H.R. Horvitz. 1988. A genetic pathway for the development of the Caenorhabditis elegans HSN motor neurons. Nature 336: 638-646.

Duncan, I. 1986. Control of bithorax complex functions by the 
segmentation gene fushi-tarazu of Drosophila melanogaster. Cell 47: 297-309.

Finney, M. and G. Ruvkun. 1990. The unc-86 gene product couples cell lineage and cell identity in C. elegans. Cell 63: 895905.

Francis, R. and R.H. Waterston. 1991. Muscle cell attachment in Caenorhabditis elegans. J. Cell Biol. 114: 465-479.

Gibson, G. and W.J. Gehring. 1988. Head and thoracic transformation caused by ectopic expression of Antennapedia during Drosophila development. Development 102: 657-675.

González-Reyes, A. and G. Morata. 1990. The developmental effect of overexpressing a $U b x$ product in Drosophila embryos is dependent on its interactions with other homeotic products. Cell 61: 515-522.

González-Reyes, A., N. Urquia, W.J. Gehring, G. Struhl, and G. Morata. 1990. Are cross-regulatory interactions between homoeotic genes functionally significant? Nature 344: 78-80.

Hedgecock, E.M., J.E. Sulston, and J.N. Thomson. 1983. Mutations affecting programmed cell deaths in the nematode Caenorhabditis elegans. Science 220: 1277-1279.

Hedgecock, E.M., J. Culotti, D. Hall, and B. Stern. 1987. Genetics of cell and axon migrations in Caenorhabditis elegans. Development 100: 365-382.

Ingham, P. and A. Martinez-Arias. 1986. The correct activation of Antennapedia and bithorax complex genes requires the fushi-tarazu gene. Nature 324: 592-597.

Jürgens, G. 1988. Head and tail development of the Drosophila embryo involves spalt, a novel homeotic gene. EMBO J. 7: 189-196.

- 1987. Segmental organization of the tail region in the embryo of Drosophila melanogaster. A blastoderm fate map of the cuticle structures of the larval tail region. Wilhelm Roux's Arch. Dev. Biol. 196: 141-157.

Karch, F., W. Bender, and B. Weiffenbach. 1990. abdA expression in Drosophila embryos. Genes \& Dev. 4: 1573-1587.

Kaufman, T.C., M.A. Seeger, and G. Olsen. 1990. Molecular and genetic organization of the Antennapedia gene complex of Drosophila melanogaster. Adv. Genet. 27: 309-362.

Kenyon, C. 1986. A gene involved in the development of the posterior body region of Caenorhabditis elegans. Cell 46: 477-487.

Kenyon, C. and B. Wang. 1991. A cluster of Antennapedia-class homeobox genes in a nonsegmented animal. Science 253: 516-517.

Kuziora, A. and W. McGinnis. 1988. Autoregulation of a Drosophila homeotic selector gene. Cell 55: 477-485.

Lamka, M.L., A.M. Boulet, and S. Sakonju. 1992. Ectopic expression of UBX and ABD-B proteins during Drosophila embryogenesis: Competition, not a functional hierarchy, explains phenotypic suppression. Development 116: 841-854.

Loer, C.M. and C. Kenyon. 1993. Serotonin-deficient mutants and male mating behavior in the nematode C. elegans. $I$. Neurosci., in press.

Macías, A., J. Casanova, and G. Morata. 1990. Expression and regulation of the $a b d-A$ gene of Drosophila. Development 110: 1197-1207.

Mann, R.S. and D.S. Hogness. 1990. Functional dissection of Ultrabithorax proteins in D. melanogaster. Cell 60: 597610.

McGinnis, W. and R. Krumlauf. 1992. Homeobox genes and axial patterning. Cell 68: 283-302.

Salser, S.J. and C. Kenyon. 1992. Activation of a C. elegans Antennapedia homolog in migrating cells controls their direction of migration. Nature 355: 255-258.

Schaller, D., C. Wittmann, A. Spicher, F. Muller, and H. Tobler. 1990. Cloning and analysis of three new homeobox genes from the nematode Caenorhabditis elegans. Nucleic Acids Res. 18: 2033-2036.

Stringham, E.G., D.K. Dixon, D. Jones, and E.P. Candido. 1992. Temporal and spatial expression patterns of the small heatshock (hsp 16) genes in transgenic Caenorhabditis elegans. Mol. Biol. Cell 3: 221-233.

Struhl, G. 1982. Genes controlling segmental specification in the Drosophila thorax. Proc. Natl. Acad. Sci. 79: 7380-7384.

Struhl, G. and R.A.H. White. 1985. Regulation of the Ultrabithorax gene of Drosophila by other bithorax complex genes. Cell 43: 507-519.

Sulston, J.E. and H.R. Horvitz. 1977. Post-embryonic cell lineages of the nematode Caenorhabditis elegans. Dev. Biol. 56: 110-156.

Wang, B., M. Müller-Immergluck, J. Austin, N.T. Robinson, A. Chisholm, and C. Kenyon. 1993. A homeotic gene cluster patterns the anteroposterior body axis of C. elegans. Cell 74: $29-42$.

White, R.A.H. and M. Wilcox. 1985. Distribution of Ultrabithorax protein in Drosophila. EMBO J. 4: 2035-2043.

Wirtz, J., L.I. Fessler, and W.J. Gehring. 1986. Localization of the Antennapedia protein in Drosophila embryos and imaginal discs. EMBO J. 5: 3327-3334.

Wood, W.B., ed. 1988. The nematode Caenorhabditis elegans. Cold Spring Harbor Laboratory, Cold Spring Harbor, New York.

Zarkower, D. and J. Hodgkin. 1992. Molecular analysis of the $C$. elegans sex-determining gene tra-1: A gene encoding two zinc finger proteins. Cell 70: 237-249. 


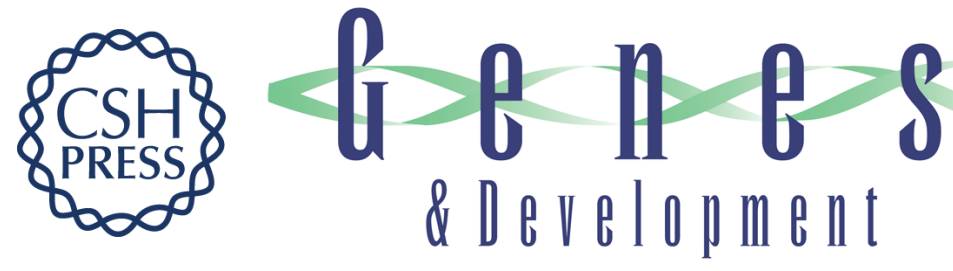

\section{Multiple HOM-C gene interactions specify cell fates in the nematode central nervous system.}

S J Salser, C M Loer and C Kenyon

Genes Dev. 1993, 7:

Access the most recent version at doi:10.1101/gad.7.9.1714

References This article cites 41 articles, 14 of which can be accessed free at:

http://genesdev.cshlp.org/content/7/9/1714.full.html\#ref-list-1

License

Email Alerting

Service

Receive free email alerts when new articles cite this article - sign up in the box at the top right corner of the article or click here.

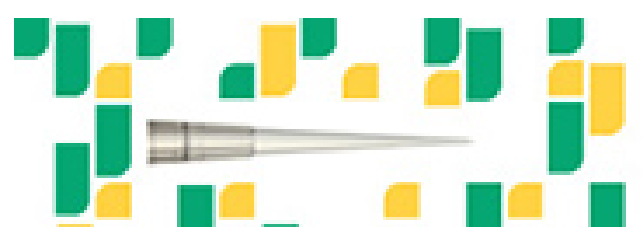

Focused on your science. 